\title{
An Accurate Closed-Form Approximation of the Energy Efficiency-Spectral Efficiency Trade-Off over the MIMO Rayleigh Fading Channel
}

\author{
Fabien Héliot, Oluwakayode Onireti,and Muhammad Ali Imran \\ Centre for Communication Systems Research (CCSR), Faculty of Electronics \& Physical Sciences, \\ University of Surrey, Guildford GU2 7XH, UK. Email: F.Heliot@surrey.ac.uk
}

\begin{abstract}
Energy efficiency (EE) is gradually becoming one of the key criteria, along with the spectral efficiency (SE), for evaluating communication system performances. However, minimizing the EE while maximizing the $\mathrm{SE}$ are conflicting objectives and, thus, the main criterion for designing efficient communication systems will become the trade-off between SE and EE. The EESE trade-off for the multi-input multi-output (MIMO) Rayleigh fading channel has been accurately approximated in the past but only in the low-SE regime. In this paper, we propose a novel and more generic closed-form approximation of this EE-SE tradeoff which exhibits a greater accuracy for a wider range of SE values and antenna configurations. Our expression, which can easily be used for evaluating and comparing the EE-SE tradeoff of MIMO communication system, has been utilized in this paper for analyzing the impact of using multiple antennas on the EE and the EE gain of MIMO in comparison with singleinput single-output (SISO) system. Our results indicate that EE can be improved predominantly through receive diversity in the low-SE regime and that MIMO is far more energy efficient than SISO at high SE over the Rayleigh fading channel.
\end{abstract}

\section{INTRODUCTION}

In the current context of growing energy demand and increasing energy price, energy efficiency (EE) is gradually becoming a key criterion in the design of communication networks. So far, the main design criterion was the spectral efficiency (SE) and as a result the energy consumption issues have received little, if any attention, e.g. development of $3 \mathrm{G}$ systems.

The SE, as a metric, is traditionally used for evaluating and comparing the efficiency of communication systems. It indicates how efficiently a limited frequency spectrum is utilized and is usually expressed in terms of bits/s/Hz. However, it does not provide any insight on how efficiently the energy is consumed within a system. In order to include this aspect in the performance evaluation framework, a metric that takes into account the energy consumption is required. Such a metric, the bit-per-Joule capacity (bits/J) has first been introduced in [1] and is simply defined as the ratio of the capacity to the rate of energy expenditure, i.e. to the signal power. It can be seen as a special case of the capacity per unit cost, which has been defined in [2]. In the context of energy limited wireless network, it can also be interpreted as the maximum amount of bits that can be delivered by the network divided by the energy in Joule that is utilized for delivering these bits [3]. Other metrics for EE have also been considered in the literature such as the rate per energy [4], the Joule-per-bit [5] and the more traditional energy-per-bit to noise power spectral density ratio, i.e. $E_{b} / N_{0}$, [1], [6]-[8].

In the future mobile systems, e.g. Long Term EvolutionAdvanced (LTE-A), the energy consumption will have to be taken into account for reducing the ever increasing running costs of the networks while keeping an acceptable SE. However, minimizing the EE while maximizing the SE are conflicting objectives. Consequently, the trade-off between $\mathrm{SE}$ and EE will become the main criterion for designing efficient communication systems. In the past, researches on the EE-SE trade-off have mainly been driven by power-limited applications, such as underwater acoustic telemetry [1] and wireless ad-hoc networks [5]. Nowadays, these studies are being extended to the area of unlimited power systems, e.g. cellular networks (base/relay stations), due to the the global aspiration for energy saving and reduction of carbon footprint.

The concept of power-bandwidth trade-off has first been introduced in [6], where an approximation of the EE-SE tradeoff has been derived for the additive white Gaussian noise (AWGN), colored noise and multi-input multi-output (MIMO) fading channels based on the first and second derivatives of the channel capacity. This linear approximation is accurate in the low-SE regime but largely inaccurate otherwise. This work has inspired numerous other works where the same analytical method was used to approximate the EE-SE tradeoff of correlated multi antenna [7], multi-user [9] and cooperative communication [10]-[12] systems in the low-SE regime. Moreover, closed-form expressions of the EE-SE trade-off have been derived in [1] and in [6] for the AWGN channel and the deterministic channel with colored noise, respectively.

In this paper, we derive a novel and generic closedform approximation of the EE-SE trade-off over the MIMO Rayleigh fading channel and demonstrate its great accuracy for numerous antenna configurations and a wider range of $\mathrm{SE}$ values than the approximation in [6]. In Section II, we recall the classic point-to-point MIMO system model and introduce the two main approaches that can be found in the literature for deriving a closed-form expression of the Ergodic MIMO channel capacity. In Section III, we first derive an accurate closed-form approximation of the EE-SE trade-off in the case that the MIMO channel has an equal number of transmit and receive antennas. Furthermore, we extend our 
derivation for a wide range of antenna settings by designing a parametric function through the use of a heuristic curve fitting method [13]-[16]. Numerical results show the great accuracy of our approximation for a wide range of spectral efficiency values and numerous antenna configurations. In Section IV, we utilize our closed-form approximation for analyzing the EE of MIMO system in low-SE regime and the impact of multiple antennas on the EE. Results show that EE can be improved mainly through receive diversity in the low-SE regime and that MIMO system is far more energy efficient than singleinput single-output (SISO) system over a Rayleigh fading channel, especially at high SE. Finally, conclusions are drawn in Section V.

\section{Classic MiMO System Model}

We consider a classic MIMO communication system where a signal $\mathbf{x} \in \mathbb{C}^{t \times 1}$ is transmitted over $t$ transmit antennas and is received by $r$ receive antennas as

$$
\mathbf{y}=\mathbf{H x}+\mathbf{n},
$$

where $\mathbf{H} \in \mathbb{C}^{r \times t}$ and $\mathbf{n} \in \mathbb{C}^{r \times 1}$ characterize the MIMO channel and the AWGN noise, respectively. In this regards, we assume that $\mathbf{H}$ is a random matrix having independent and identically distributed (i.i.d.) complex circular Gaussian entries with zero-mean and unit variance, and that $\mathbf{n}$ belongs to an $r$-dimensional complex zero-mean circular symmetric Gaussian distribution with variance $N$ per dimension, i.e. $\mathbf{n} \sim \mathcal{N}_{c}\left(\mathbf{0}_{r}, N \mathbf{I}_{r}\right)$, where $\mathbf{0}_{r}$ and $\mathbf{I}_{r}$ denote the all-zero matrix and the identity matrix, respectively, of dimension $r \times r$. In addition, we consider that $\mathbf{x} \sim \mathcal{N}_{c}\left(\mathbf{0}_{t},(P / t) \mathbf{I}_{t}\right)$, where $P$ is the average power of the transmit signal such that $\operatorname{tr}\left(\mathrm{E}\left\{\mathbf{x x}^{\dagger}\right\}\right)=P, \operatorname{tr}($.$) and \mathrm{E}\{$.$\} stand for the trace$ and expectation. The Ergodic capacity of the MIMO Rayleigh channel is accordingly expressed as [17]

$$
C \triangleq W \mathrm{E}_{\mathbf{H}}\left\{\log _{2}\left|\mathbf{I}_{r}+\frac{\gamma}{t} \mathbf{H H}^{\dagger}\right|\right\}
$$

where $W$ is the bandwidth, $\gamma \triangleq P / N$ is the average signalto-noise ratio (SNR) and $|$.$| is the determinant.$

In the literature, two main approaches have been followed for deriving either closed-form expressions or approximations of the Ergodic capacity as in (2). In [17], the expression of $C$ has been simplified into an analytical formula by computing the expectation of the ordered eigenvalues of the Wishart matrix $\mathcal{W} \triangleq \mathbf{H H}^{\dagger}$ or $\mathbf{H}^{\dagger} \mathbf{H}$ if $r<t$ or $r \geq t$, respectively. This work has sparkled a flurry of interest in finding proper closedform expressions of the Ergodic capacity [18]-[20]. In Parallel, a closed-form approximation of (2) has been derived in [21] by relying on asymptotical analysis and random matrix theory. The former approach yields perfectly accurate expressions but at the expense of cumbersome formulations. Whereas the later approach is less accurate, especially for small values of $r$ and $t$, but with a far simpler formulation. Consequently, we use the closed-form approximation in [21], which is given by $C \approx$

$$
\widetilde{C}=-\frac{W t}{\ln (2)}\left[(1+\beta) \ln (w)+q_{0} r_{0}+\ln \left(r_{0}\right)+\beta \ln \left(\frac{q_{0}}{\beta}\right)\right],
$$

as a starting point for deriving our accurate closed-form approximation of the EE-SE trade-off for MIMO Rayleigh fading channel. Note that in (3), $w=1 / \sqrt{\gamma}, \beta=r / t$, and

$$
\begin{aligned}
& q_{0}=\frac{\beta-1-w^{2}+\sqrt{\left(\beta-1-w^{2}\right)^{2}+4 w^{2} \beta}}{2 w} \\
& r_{0}=\frac{1-\beta-w^{2}+\sqrt{\left(1-\beta-w^{2}\right)^{2}+4 w^{2}}}{2 w} .
\end{aligned}
$$

This accurate approximation has been derived by assuming large number of antennas $t$ and $r$, however, its accuracy has been deemed acceptable even for small number of antennas, as it is explained in [21].

Let us now define $\bar{q}_{0}=2 q_{0} / w+1$ and $\bar{r}_{0}=2 r_{0} / w+1$, then, the term $(1+\beta) \ln (w)+\ln \left(r_{0}\right)+\beta \ln \left(\frac{q_{0}}{\beta}\right)$ in (4) simplifies as $\ln (2)-\ln \left(1+\bar{q}_{0}\right)+\beta\left(\ln (2)-\ln \left(1+\bar{r}_{0}\right)\right)$. In addition, the term $q_{0} r_{0}$ in (4) simplifies as $1 / 2-1 /\left(1+\bar{q}_{0}\right)+\beta\left(1 / 2-1 /\left(1+\bar{r}_{0}\right)\right)$. Thus, equation (4) can be re-expressed as

$$
\widetilde{C}=\frac{W}{\ln (2)}\left(S_{t}+S_{r}\right)=\frac{W \bar{S}}{\ln (2)},
$$

where $\bar{S}=S_{t}+S_{r}$; moreover, $S_{t}$ and $S_{r}$ are given by

$$
\begin{aligned}
& S_{t}=t\left(-\frac{1}{2}-\ln (2)+\frac{1}{\left(1+\bar{q}_{0}\right)}+\ln \left(1+\bar{q}_{0}\right)\right) \text { and } \\
& S_{t}=r\left(-\frac{1}{2}-\ln (2)+\frac{1}{\left(1+\bar{r}_{0}\right)}+\ln \left(1+\bar{r}_{0}\right)\right),
\end{aligned}
$$

respectively.

\section{Closed-Form APPROXIMATION OF THE EE-SE TRADE-OFF}

The concept of EE-SE trade-off can easily be illustrated by using the Shannon's capacity theorem. For instance, the AWGN channel single-user capacity can be expressed as

$C=W f(\gamma)=W \log _{2}(1+\gamma)=W \log _{2}\left(1+\frac{P}{N_{0} W}\right) \geq R$,

where $R$ is the achievable rate of an encoder in bits per second. This rate $R$ is dependent of the power $P$, the bandwidth $W$ and the noise spectral density $N_{0}$. The SE $S$ is usually defined as the ratio of the rate $R$ to the bandwidth $W$. Whereas the EE can be either expressed in terms of $E_{b} / N_{0}$ or $C_{J} ; E_{b}$ is the energy-per-bit in Joule per bits and is equivalent to $P / R ; C_{J}$ is the bit-per-Joule capacity and is equivalent to $1 / E_{b}$. Using the inverse function of $f, f^{-1}$, for expressing $\gamma$ as a function of $R$ and inserting $P=R E_{b}$ in (7), the following inequalities are obtained [1]

$$
\gamma \geq f^{-1}(S) \Leftrightarrow \begin{aligned}
& \frac{E_{b}}{N_{0}} \geq \frac{2^{S}-1}{S} \\
& C_{J} \leq \frac{S}{N_{0}\left(2^{S}-1\right)}
\end{aligned},
$$

where $S=R / W$. Equation (8) clearly demonstrates the existence of a trade-off between the $\mathrm{EE}$ and SE for the rate $R$ to be achieved. Moreover, this trade-off can be expressed into a closed-form for the AWGN channel. 
The previous example for the AWGN channel explicitly shows that the problem of finding a closed-form expression for the EE-SE trade-off boils down to obtaining a closed-form expression for the inverse function $f^{-1}(S)$. Coming back to the MIMO Rayleigh fading case, we can re-expressed the first equation in (6) as

$$
g_{t}\left(S_{t}\right)=-\frac{1}{\left(1+\bar{q}_{0}\right)} \exp \left(-\frac{1}{1+\bar{q}_{0}}\right)
$$

where $g_{t}\left(S_{t}\right)=-\exp \left(-\left(\frac{S_{t}}{t}+\frac{1}{2}+\ln (2)\right)\right)$. The Lambert $W$ function is the inverse function of $f(w)=w \exp (w)$ and, thus, it satisfies $W(z) e^{W(z)}=z$, where $w, z \in \mathbb{C}$ [22]. In addition, the real branch of the Lambert function, which is denoted by $W_{0}$, is such that $W_{0}:[-\exp (-1),+\infty) \mapsto$ $[-1,+\infty)$. Knowing that $S_{t} \in \mathbb{R}^{+}$, it implies that $g_{t}\left(S_{t}\right) \in$ $\left[-\frac{1}{2} e^{-\frac{1}{2}}, 0\right]$ belongs to the domain of $W_{0}$. Consequently, we can reformulate (9) as

$$
\begin{aligned}
-\frac{1}{\left(1+\bar{q}_{0}\right)} & =W_{0}\left(g_{t}\left(S_{t}\right)\right), \\
\bar{q}_{0} & =-\left[1+\frac{1}{W_{0}\left(g_{t}\left(S_{t}\right)\right)}\right] .
\end{aligned}
$$

Similarly, $\bar{r}_{0}=-\left[1+\frac{1}{W_{0}\left(g_{r}\left(S_{r}\right)\right)}\right]$. Furthermore, it can easily be demonstrated that

$$
\bar{q}_{0} \bar{r}_{0}=1+2 \gamma(1+\beta)
$$

which lead us to the following expression

$$
\gamma=\frac{-1+\left[1+\frac{1}{W_{0}\left(g_{t}\left(S_{t}\right)\right)}\right]\left[1+\frac{1}{W_{0}\left(g_{r}\left(S_{r}\right)\right)}\right]}{2(1+\beta)} .
$$

\section{A. Case of $t=r$}

In the case that $t=r, \beta=1$ and $q_{0}=r_{0}$ according to (4), hence, $S_{t}=S_{r}=\bar{S} / 2$. Moreover, knowing that $\bar{S}=$ $\frac{\widetilde{C} \ln (2)}{W} \geq S \ln (2)$, we obtain our closed-form approximation of the EE-SE trade-off by inserting $S_{t}=S_{r}=S \ln (2) / 2$ in (12) as follows

$$
\frac{E_{b}}{N_{0}} \geq \frac{-1+\left[1+\left(W_{0}\left(-2^{-\left(\frac{S}{2 r}+1\right)} e^{-\frac{1}{2}}\right)\right)^{-1}\right]^{2}}{4 S} .
$$

\section{B. Case of $t \neq r$}

In this case, the problem of defining a closed-form for the EE-SE trade-off is equivalent to expressing both $S_{t}$ and $S_{r}$ as a function of $\bar{S}$ in (12). Indeed, we defined $\bar{S}$ as $\bar{S}=S_{r}+S_{t}$ in (5) and, thus, if we could define a parametric function $\Phi_{t, r}(\bar{S})$ such that $\Phi_{t, r}(\bar{S}) \approx S_{r}-S_{t}$, then, we would obtain $S_{t}$ and $S_{r}$ as a function of solely $t, r$ and $\bar{S}$ by solving a simple system of linear equations. The difference $S_{r}-S_{t}$ can be re-expressed as

$$
\Phi_{t, r}(\bar{S}) \approx S_{r}-S_{t}=\ln \left(\frac{2^{t}\left(1+\bar{r}_{0}\right)^{r}}{2^{r}\left(1+\bar{q}_{0}\right)^{t}}\right),
$$

since it can easily be proved that $r\left(-1 / 2+1 /\left(1+\bar{r}_{0}\right)\right)-$ $t\left(-1 / 2+1 /\left(1+\bar{q}_{0}\right)\right)=0$. In addition, we define $\phi_{t, r}(\bar{S})=$ $e^{\frac{\Phi_{t, r}(\bar{S})}{r}} \approx e^{\frac{S_{r}-S_{t}}{r}}$.

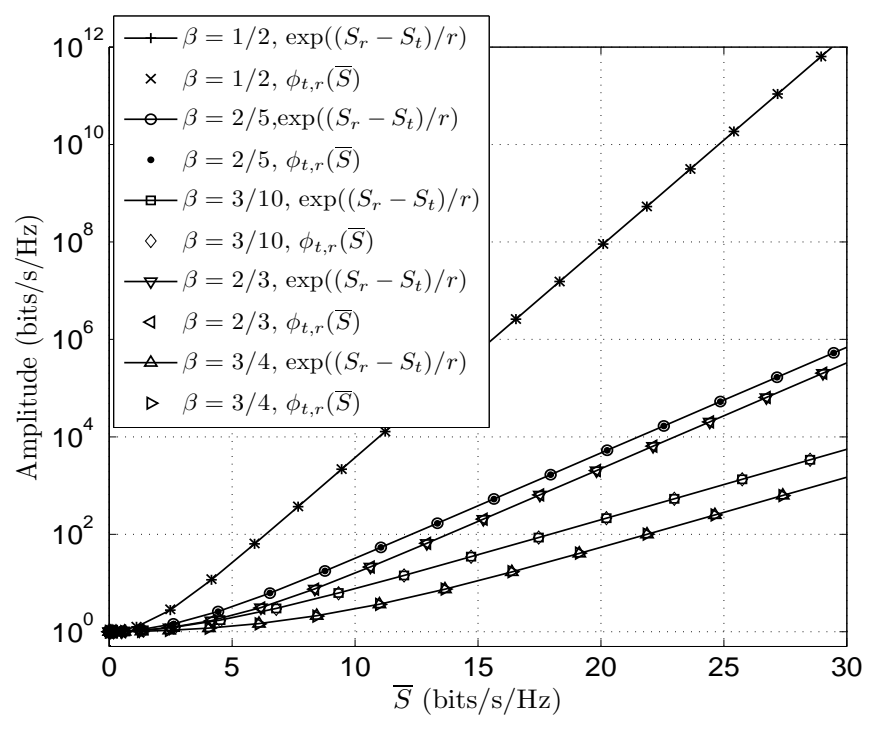

Fig. 1. Comparison of $e^{\frac{S_{r}-S_{t}}{r}}$ with $\phi_{t, r}(\bar{S})$ for various $\beta$ values.

In the heuristic curve fitting method proposed in [13], a parametric function is designed in terms of elementary functions and three independent parameters for solving a curve fitting problem. In this paper, we use this curve fitting method to design the parametric function $\phi_{t, r}(\bar{S})$ that tightly fits $e^{\frac{S_{r}-S_{t}}{r}}$ for $\left.\beta \in \mathbb{B}_{0}=\{p / q \mid p \in[1, \ldots, 9], q \in[2, \ldots, 10]\}, q>p\right\}$, i.e. $\beta<1$, and $\bar{S}$ values between 0 and $30 \mathrm{bits} / \mathrm{s} / \mathrm{Hz}$. We first numerically evaluated $e^{\frac{S_{r}-S_{t}}{r}}$ as a function of $\bar{S}$ for different values of $\beta$ and then collected the resulting curves in Fig. 1. It can be noticed that $e^{\frac{S_{r}-S_{t}}{r}}$ presents the feature of an exponential function at low $\bar{S}$ and of a linear function at high $\bar{S}$ (in logarithmic scale). In addition, this function is monotonic and is value at $\bar{S}=0$ is one. In the effort of obtaining the function that best fits the curves of Fig. 1, the curve fitting method leads to the parametric function

$$
\phi_{t, r}(\bar{S})=1+\eta_{0}\left[\cosh \left(\bar{S} /\left(r \eta_{2}\right)\right)^{\eta_{1}}-1\right],
$$

which provides a satisfying approximation, as it is illustrated in Fig. 1 for $\beta=1 / 2,2 / 5,3 / 10,2 / 3,3 / 4, \eta_{0}=$ $1,1,1,0.134,0.093, \eta_{1}=2.55,2.247,1.988,0.412,0.37$, and $\eta_{2}=2.55,2.247,1.988,0.412,0.367$, respectively. Moreover, we have observed that $\eta_{0}=1$ and $\eta_{2}=\eta_{1}$ when $\beta \leq 0.5$ such that $\phi_{t, r}(\bar{S})$ simplifies as $\phi_{t, r}(\bar{S})=\cosh \left(\bar{S} /\left(r \eta_{1}\right)\right)^{\eta_{1}}$ in this case.

Consequently, we obtain that

$$
\begin{aligned}
& \Phi_{t, r}(\bar{S})=r \eta_{1} \ln \left(\cosh \left(\bar{S} /\left(r \eta_{1}\right)\right)\right) \\
& \Phi_{t, r}(\bar{S})=r \ln \left(1+\eta_{0}\left[\cosh \left(\bar{S} /\left(r \eta_{2}\right)\right)^{\eta_{1}}-1\right]\right)
\end{aligned}
$$

are an appropriate choice of parametric functions for tightly approximate $S_{r}-S_{t}$ as a function of $\bar{S}$ when $\beta \leq 0.5$ and $0.5<\beta<1$, respectively. The tightness of $\Phi_{t, r}(\overline{\bar{S}})$ can be controlled by adjusting the parameters $\eta_{0}, \eta_{1}$ and $\eta_{2}$ in (16) such that the following mean squared error (MSE) equation is 


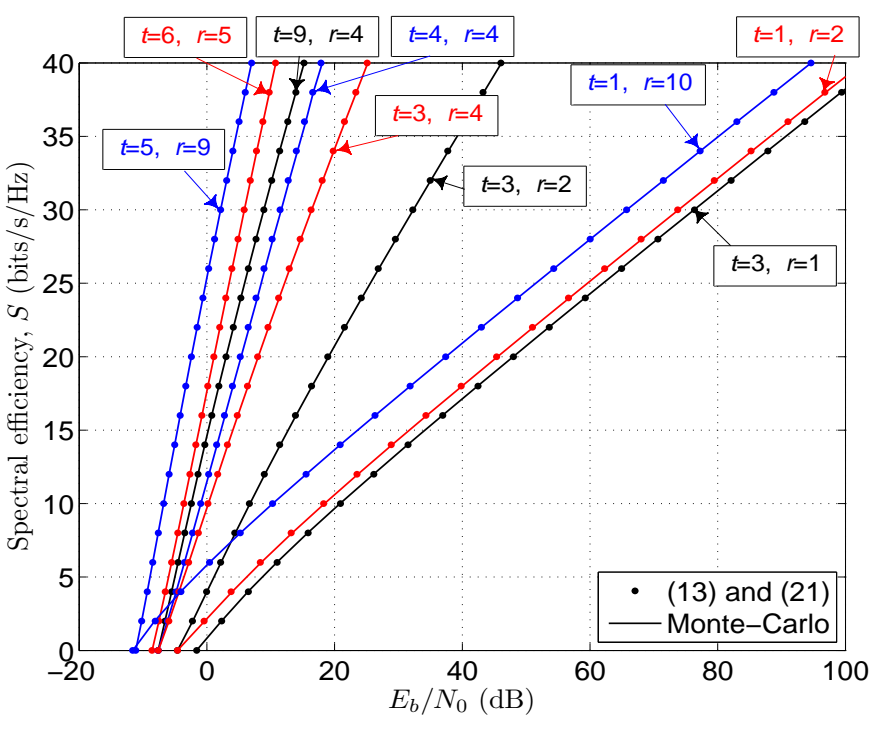

Fig. 2. Comparison of our closed-form approximations in (13) and (21) with Monte-Carlo simulation for various $t$ and $r$ values.

minimized, i.e. $\varepsilon_{0} \ll 1$, when $\beta<1$

$$
\frac{1}{10 N+1} \sum_{\bar{S}=0}^{N}\left|\left(S_{r}-S_{t}\right)-\Phi_{t, r}(\bar{S})\right|^{2} \leq \varepsilon_{0} .
$$

Using (17), we have obtained the coefficients $\eta_{0}, \eta_{1}$ and $\eta_{2}$, which are collected in Table I, for $\beta \in \mathbb{B}_{0}, N=30$, $\varepsilon_{0}=$ $1 \times 10^{-3}$ and with an incremental step of $0.1 \mathrm{bits} / \mathrm{s} / \mathrm{Hz}$ for $\bar{S}$. Similarly, we utilize the same process for designing the parametric function $\phi_{r, t}(\bar{S})$ that tightly fits $e^{\frac{S_{t}-S_{r}}{t}}$ for $\beta \in$ $\left.\mathbb{B}_{1}=\{p / q \mid p \in[2, \ldots, 10], q \in[1, \ldots, 9]\}, p>q\right\}$, i.e. $\beta>$ 1 , and $\bar{S}$ values between 0 and $30 \mathrm{bits} / \mathrm{s} / \mathrm{Hz}$. Consequently, we obtain that

$$
\begin{aligned}
& \Phi_{r, t}(\bar{S})=t \ln \left(1+\eta_{0}\left[\cosh \left(\bar{S} /\left(t \eta_{2}\right)\right)^{\eta_{1}}-1\right]\right) \\
& \Phi_{r, t}(\bar{S})=t \eta_{1} \ln \left(\cosh \left(\bar{S} /\left(t \eta_{1}\right)\right)\right)
\end{aligned}
$$

are an appropriate choice of parametric functions for tightly approximate $S_{t}-S_{r}$ as a function of $\bar{S}$ when $1<\beta<2$ and $\beta \geq 2$, respectively, where $\eta_{0}, \eta_{1}$ and $\eta_{2}$ are given in Table I.

Finally, we obtain $S_{t}$ and $S_{r}$ as a function of solely $t, r$ and $\bar{S}$ by using (16) and (18) with $\bar{S}=S_{r}+S_{t}$ such that

$$
\begin{aligned}
& S_{t}=\left\{\bar{S}-\zeta \alpha \ln \left(1+\eta_{0}\left[\cosh \left(\bar{S} /\left(\alpha \eta_{2}\right)\right)^{\eta_{1}}-1\right]\right)\right\} / 2 \\
& S_{r}=\left\{\bar{S}+\zeta \alpha \ln \left(1+\eta_{0}\left[\cosh \left(\bar{S} /\left(\alpha \eta_{2}\right)\right)^{\eta_{1}}-1\right]\right)\right\} / 2
\end{aligned},
$$

for $0.5<\beta<2, \beta \neq 1$, where $\alpha=\min (t, r), \zeta=$ $-\operatorname{sign}(\ln (\beta))$ and $\operatorname{sign}(x)=-1$ or 1 if $x<0$ or $x>0$, respectively. In addition, $S_{t}$ and $S_{r}$ can be formulated as

$$
\begin{aligned}
& S_{t}=\delta(\zeta+1) \bar{S}+\zeta \frac{\alpha \eta_{1}}{2} \ln \left(\frac{2}{1-e^{\frac{-2 \bar{S}}{\alpha \eta_{1}}}}\right), \\
& S_{r}=\delta(\zeta-1) \bar{S}-\zeta \frac{\alpha \eta_{1}}{2} \ln \left(\frac{2}{1-e^{\frac{-2 \bar{S}}{\alpha \eta_{1}}}}\right)
\end{aligned}
$$

when $\beta \leq 0.5$ or $\beta \geq 2$, where $\delta(x)=1$ or 0 if $x=0$ or $x \neq 0$, respectively. Our closed-form approximation of the

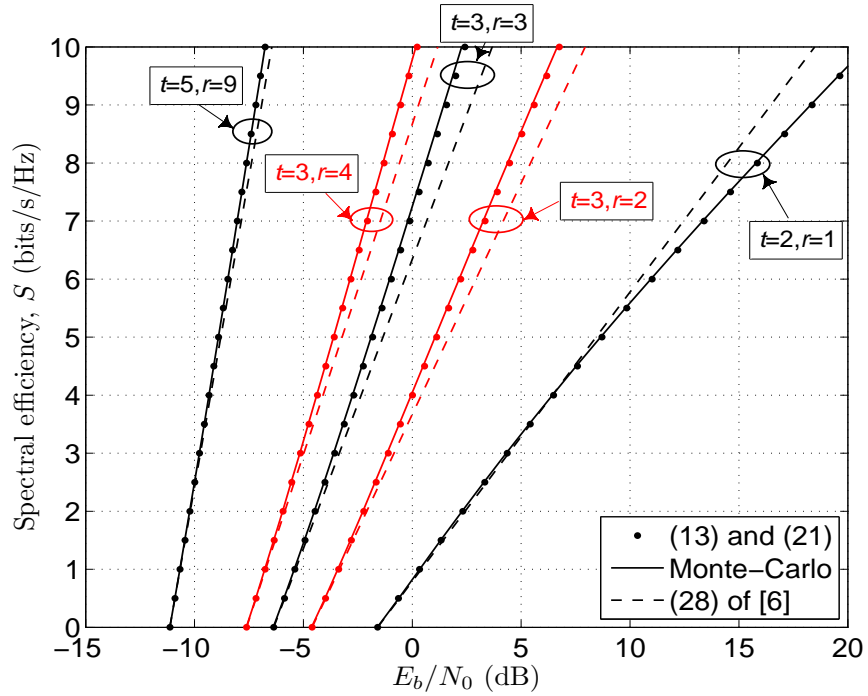

Fig. 3. Comparison of our closed-form approximations in (13) and (21) with the approximation in [6] and Monte-Carlo simulation for various $t$ and $r$ values.

EE-SE trade-off is eventually expressed by inserting $S_{t}$ and $S_{r}$ (equations (19) or (20)) in (12) for $\bar{S} \geq=S \ln (2)$ such that

$$
\frac{E_{b}}{N_{0}} \geq \frac{-1+\left[1+\frac{1}{W_{0}\left(g_{t}\left(S_{t}\right)\right)}\right]\left[1+\frac{1}{W_{0}\left(g_{r}\left(S_{r}\right)\right)}\right]}{2 S(1+\beta)},
$$

for any $\beta \in \mathbb{B}_{0} \cup \mathbb{B}_{1}$.

In Figs. 2 and 3, we compare our closed-form approximation, i.e. equations (13) and (21), with the approximation method of [6] and Monte-Carlo simulation for various $t$ and $r$ values. In [6], it has been demonstrated that the EE of a system depends mainly on the SE in the low-power/low-SE regime such that the EE-SE trade-off can be approximated as (equation (28) of [6])

$$
10 \log _{10}\left(\frac{E_{b}}{N_{0}}\right) \approx 10 \log _{10}\left({\frac{E_{b}}{N_{0}}}_{\min }\right)+\frac{S}{S_{0}} 10 \log _{10}(2) .
$$

where $\frac{E_{b}}{N_{0} \text { min }}=\frac{\ln (2)}{\dot{S}(0)}$ and $S_{0}=\frac{2[\dot{S}(0)]^{2}}{-\dot{S}(0)}$ are the minimum energy per bit and the slope of the SE, respectively, and $\dot{S}(0)$ and $\ddot{S}(0)$ are the first and second order derivatives of $S(\gamma)$ when $\gamma=0$. In addition, $\dot{S}(0)=r$ and $S_{0}=\frac{2 t r}{t+r}$, i.e. equations (213) and (215) of [6], in the MIMO rayleigh fading case when $\mathbf{H}$ is unknown and equal power allocation is assumed at the transmitter. The results clearly demonstrate the tight fitness between Monte-Carlo simulation and our closed-form approximation, hence, they graphically show the accuracy of the latter. The results also indicate that the closedform approximation in [6] is only accurate for low SE, i.e. 1 to 5 bits/s/Hz in Fig. 3, and its accuracy depends on the antenna configuration, whereas, our closed-form approximation is accurate for any antenna settings such that $\beta \in \mathbb{B}_{0} \cup \mathbb{B}_{1} \cup\{1\}$ and for $S$ values up to $30 / \ln (2) \mathrm{bits} / \mathrm{s} / \mathrm{Hz}$. 


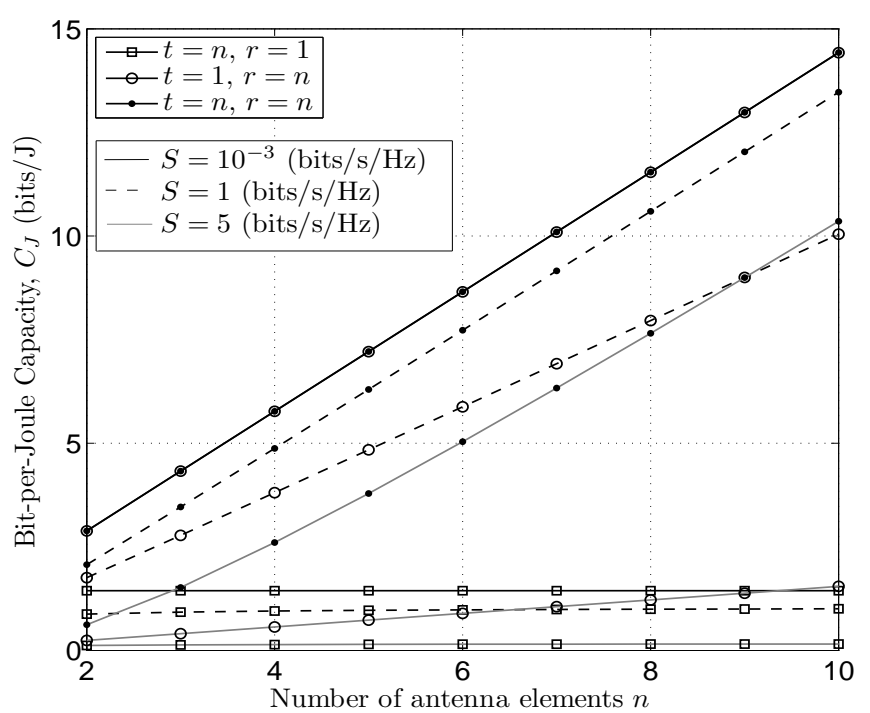

Fig. 4. Impact of the number of antennas on the EE.

\section{ENERGy EFFICIENCY ANALYSis OF MIMO System}

In this section, we use our closed-form approximation of the EE-SE trade-off for analyzing the EE of MIMO system at low SE-regime, the impact of the antenna configuration on the EE and the EE gain of MIMO in comparison with SISO system.

By using our closed-form approximation of the EE-SE trade-off, we can cross-validate the result in (213) of [6], i.e. the minimum energy per bit of a MIMO system over the Rayleigh fading channel is $\ln (2) / r$. Let us assume that $S \sim 0$ in (13), it then implies that (13) can be re-expressed as

$$
\frac{E_{b}}{N_{0}} \geq \frac{-1+\left[1+\left(-\frac{1}{2}\left(1-\frac{S \ln (2)}{r}\right)\right)^{-1}\right]^{2}}{4 S}
$$

since $-2^{-\left(\frac{S}{2 r}+1\right)} e^{-\frac{1}{2}}=-\frac{1}{2} e^{-\frac{1}{2}\left(1+\frac{S \ln (2)}{r}\right)}=-\frac{1}{2} e^{-\frac{S \ln (2)}{r}}$ $\times e^{-\frac{1}{2}\left(1-\frac{S \ln (2)}{r}\right)}$ which in turn simplifies as $-\frac{1}{2}\left(1-\frac{S \ln (2)}{r}\right) e^{-\frac{1}{2}\left(1-\frac{S \ln (2)}{r}\right)}$ because $e^{-x} \stackrel{0}{\sim} 1-x$. Consequently, $W_{0}\left(-2^{-\left(\frac{S}{2 r}+1\right)} e^{-\frac{1}{2}}\right) \stackrel{0}{\sim}-\frac{1}{2}\left(1-\frac{S \ln (2)}{r}\right)$. After simplifying further (23), we obtain that

$$
\frac{E_{b}}{N_{0}} \geq \frac{4 r \ln (2)}{4(-r+S \ln (2))^{2}} \stackrel{0}{\sim} \frac{4 r \ln (2)}{4(-r)^{2}},
$$

which finally simplifies as

$$
\frac{E_{b}}{N_{0}} \geq \frac{\ln (2)}{r} .
$$

In Fig. 4, we utilize our closed-form approximation, i.e. equations (13) and (21), for evaluating the impact of various antenna configurations on the EE of MIMO system when $N_{0}=1$. The results first clearly show that increasing the number of transmit antennas when $r=1$ does not affect the EE. On the other hand, for a fixed number of transmit antenna, i.e. $t=1$, the EE increases linearly with the number of receive

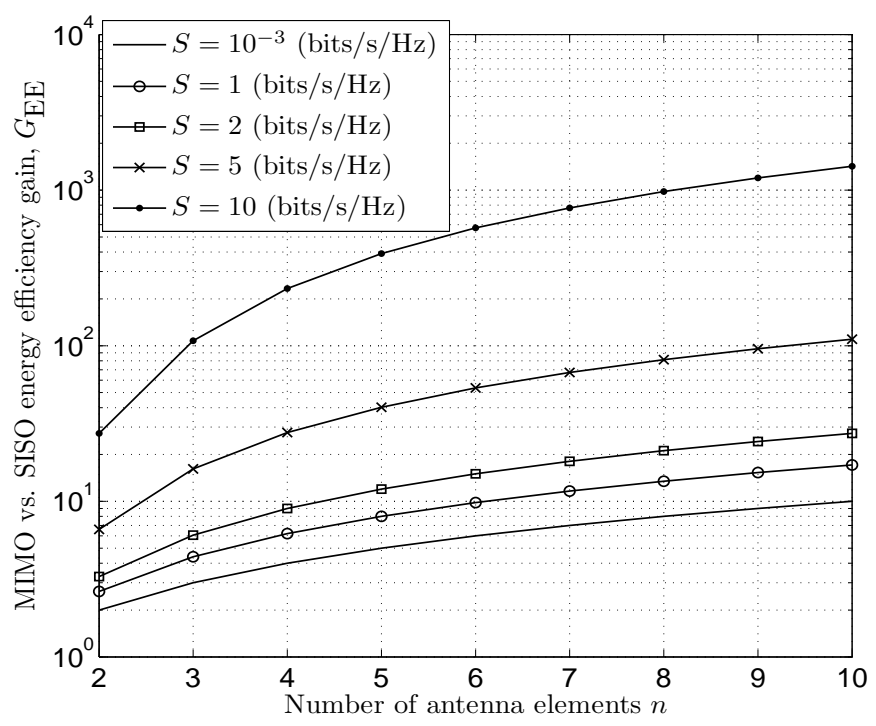

Fig. 5. EE gain of MIMO in comparison with SISO system.

antennas. Moreover, the EE of the $1 \times n$ and $n \times n$ systems are the same in the very low-SE regime, i.e. $S=10^{-3}$. Finally, it can be seen that the EE decreases when the SE increases. Overall, this graph shows that the key parameter for increasing the $\mathrm{EE}$ is the number of receive antenna $r$, which is also emphasizes in (25) since the minimum energy per bit of a MIMO system over the Rayleigh fading channel is solely dependent of $r$.

In Fig. 5, we utilize our closed-form approximation of the EE-SE trade-off for comparing the EE of MIMO against SISO system over the Rayleigh fading channel. We define the EE gain $G_{\mathrm{EE}}$ as $G_{\mathrm{EE}}=C_{J, \mathrm{MIMO}} / C_{J, \mathrm{SISO}}$, where $C_{J, \mathrm{MIMO}}$ is obtained via our closed-form approximation and $C_{J, \text { SISO }}$ is obtained through Monte-Carlo simulation. At low-SE regime, i.e. $S=10^{-3}$, we expect that $G_{\mathrm{EE}}=r$ because of (25) and this is exactly what we obtain in Fig. 5. Thus, a 10x10 MIMO system is 10 times more energy efficient than a SISO system when $S=10^{-3}$. Interestingly, as the spectral efficiency increases, as $G_{\mathrm{EE}}$ increases and, consequently, it becomes 1500 times more efficient to use a 10x10 MIMO system than a SISO system when the target $\mathrm{SE}$ is set to $10 \mathrm{bits} / \mathrm{s} / \mathrm{Hz}$. This result underlines the fact that the EE of MIMO system decreases slower than the EE of SISO as a function of SE. It clearly confirms the huge potential of MIMO for improving the EE of communication systems.

\section{CONClusion}

In this paper, an accurate closed-form approximation of the EE-SE trade-off has been derived for the MIMO Rayleigh fading channel and proved to be accurate for a wide range of spectral efficiency values and numerous antenna configurations, contrarily to the previous best-know approximation in [6]. We have first derived our approximation for the case where the MIMO channel has an equal number of transmit and receive antennas and then extended it to various other antenna 
TABLE I

PARAMETERS $\eta_{0}, \eta_{1}$ AND $\eta_{2}$ VAlues AS A FUNCTION OF $\beta$

\begin{tabular}{|c||c|c|c|c|c|c|c|c|c|c|c|c|c|c|c|c|}
\multicolumn{1}{c||}{} & \multicolumn{10}{c|}{$\beta \leq 0.5$ or $\beta \geq 2$} \\
\hline$\beta \mid 1 / \beta$ & $1 / 10$ & $1 / 9$ & $1 / 8$ & $1 / 7$ & $1 / 6$ & $1 / 5$ & $2 / 9$ & $1 / 4$ & $2 / 7$ & $3 / 10$ & $1 / 3$ & $3 / 8$ & $2 / 5$ & $3 / 7$ & $4 / 9$ & $1 / 2$ \\
\hline$\eta_{1}$ & 1.597 & 1.616 & 1.640 & 1.671 & 1.713 & 1.777 & 1.820 & 1.877 & 1.955 & 1.987 & 2.067 & 2.175 & 2.243 & 2.330 & 2.389 & 2.558 \\
\hline \multicolumn{10}{|c|}{} & \multicolumn{10}{|c|}{$0.5<\beta<1$ or $1<\beta<2$} \\
\hline$\beta \mid 1 / \beta$ & $5 / 9$ & $4 / 7$ & $3 / 5$ & $5 / 8$ & $2 / 3$ & $7 / 10$ & $5 / 7$ & $3 / 4$ & $7 / 9$ & $4 / 5$ & $5 / 6$ & $6 / 7$ & $7 / 8$ & $8 / 9$ & $9 / 10$ \\
\hline$\eta_{0}$ & 0.217 & 0.204 & 0.179 & 0.163 & 0.124 & 0.151 & 0.127 & 0.081 & 0.116 & 0.074 & 0.066 & 0.075 & 0.075 & 0.080 & 0.066 \\
$\eta_{1}$ & 0.56 & 0.54 & 0.48 & 0.46 & 0.30 & 0.57 & 0.48 & 0.26 & 0.58 & 0.32 & 0.31 & 0.53 & 0.60 & 0.74 & 0.65 & \\
$\eta_{2}$ & 0.56 & 0.54 & 0.48 & 0.46 & 0.30 & 0.55 & 0.47 & 0.26 & 0.54 & 0.31 & 0.29 & 0.47 & 0.51 & 0.60 & 0.53 \\
\hline
\end{tabular}

settings by means of an heuristic curve fitting method. The accuracy of our approximation has been shown experimentally for practical SE and antenna values. Our expression has been utilized for interpreting the EE-SE trade-off in the low-SE regime and cross-validating the minimum amount of energy that is required for transmitting bits over a MIMO Rayleigh fading channel. It has also been used for evaluating the impact of using multiple antennas on the EE. Thus, it has been pointed out that EE can be improved mainly through receive diversity in low-SE regime and that MIMO has a large potential for improving the EE of communication systems, especially in the high-SE regime. In the future, we would like to extend our method to cooperative MIMO communication and derive closed-form approximations of the EE-SE trade-off by using the works in [23] and [24] as a starting point.

\section{ACKNOWLEDGMENT}

The research leading to these results has received funding from the European Commission's Seventh Framework Programme FP7/2007-2013 under grant agreement n²47733project EARTH.

\section{REFERENCES}

[1] H. M. Kwon and T. G. Birdsall, "Channel Capacity in Bits per Joule," IEEE Jour. Oceanic Engineering, vol. OE-11, no. 1, pp. 97-99, Jan. 1986.

[2] S. Verdu, "On Channel Capacity per Unit Cost," IEEE Trans. Inf. Theory, vol. 36, no. 5, pp. 1019-1030, Sep. 1990.

[3] V. Rodoplu and T. H. Meng, "Bits-per-Joule Capacity of Energy-Limited Wireless Networks," vol. 6, no. 3, pp. 857-865, Mar. 2007.

[4] J. Gomez-Vilardebo, A. Perez-Neira, and M. Najar, "Energy Efficient Communications over the AWGN Relay Channel," IEEE Trans. Wireless Commun., vol. 9, no. 1, pp. 32-37, Jan. 2010.

[5] C. Bae and W. E. Stark, "Energy and Bandwidth Efficiency in Wireless Networks," in Proc. IEEE ICCCAS, Guilin, China, Jun. 2006.

[6] S. Verdu, "Spectral Efficiency in the Wideband Regime," IEEE Trans. Inf. Theory, vol. 48, no. 6, pp. 1319-1343, Jun. 2002.

[7] A. Lozano, A. M. Tulino, and S. Verdu, "Multiple-Antenna Capacity in the Low-Power Regime," IEEE Trans. Inf. Theory, vol. 49, no. 10, pp. 2527-2544, Oct. 2003.

[8] O. Oyman and A. J. Paulraj, "Spectral Efficiency of Relay Networks in the Power Limited Regime," in Proc. 42th Annual Allerton Conf. on Communcation, Control and Computing, Allerton, USA, Sep. 2004.
[9] G. Caire, G. Taricco, and E. Biglieri, "Suboptimality of TDMA in the Low-Power Regime," IEEE Trans. Inf. Theory, vol. 50, no. 4, pp. 608620, Apr. 2004.

[10] Y. Yao, X. Cai, and G. B. Giannakis, "On Energy Efficiency and Optimum Resource Allocation of Relay Transmissions in the Low-Power Regime," IEEE Trans. Wireless Commun., vol. 4, no. 6, pp. 2917-2927, Nov. 2005.

[11] O. Somekh, B. M. Zaidel, and S. Shamai, "Sum Rate Characterization of Joint Multiple Cell-Site Processing," IEEE Trans. Inf. Theory, vol. 53, no. 12, pp. 4473-4497, Dec. 2007.

[12] O. Simeone, O. Somekh, Y. Bar-Ness, and U. Spagnolini, "Throughput of Low-Power Cellular Systems with Collaborative Base Stations and Relaying," IEEE Trans. Inf. Theory, vol. 54, no. 1, pp. 459-467, Jan. 2008.

[13] N. C. Beaulieu and F. Rajwani, "Highly Accurate Simple Closed-Form Approximations to Lognormal Sum Distributions and Densities," IEEE Commun. Lett., vol. 8, no. 12, pp. 709-711, Dec. 2004.

[14] D. B. da Costa and M. D. Yacoub, "Average Channel Capacity for Generalized Fading Scenarios," IEEE Commun. Lett., vol. 11, no. 12, pp. 949-951, Dec. 2007.

[15] G. Fraidenraich, O. Leveque, and J. M. Cioffi, "On the MIMO Channel Capacity for the Nakagami- $m$ Channel," IEEE Trans. Inf. Theory, vol. 54 , no. 8, pp. 3752-3757, Aug. 2008.

[16] F. Héliot, M. Ghavami, and M. R. Nakhai, "An Accurate Closed-Form Approximation of the Average Probability of Error over a Log-Normal Fading Channel," IEEE Trans. Wireless Commun., vol. 7, no. 5, pp. 1495-1500, May 2008.

[17] I. E. Telatar, "Capacity of Multi-Antenna Gaussian Channels," Europ. Trans. Telecommun. and Related Technol., vol. 10, no. 6, pp. 585-596, Nov. 1999.

[18] M. Dohler, "Virtual Antenna Arrays," Ph.D. dissertation, King's College London, University of London, Nov 2003. [Online]. Available: http://perso.rd.francetelecom.fr/dohler/theses/PhD.pdf

[19] H. Shin and J. Lee, "Closed-form Formulas for Ergodic Capacity of MIMO Rayleigh Fading Channels," in ICC '03, May 2003, pp. 29963000 .

[20] M. Kang and M. Alouini, "On the Capacity of MIMO Rician Channels," in Proc. 40th Annual Allerton Conf. on Communcation, Control and Computing, Allerton, USA, Oct. 2002, pp. 936-945.

[21] E. Biglieri and G. Taricco, Transmission and Reception with Multiple Antennas: Theoretical Foundations. Now Publishers Inc., 2004.

[22] R. M. Corless, G. H. Gonnet, D. E. G. Hare, D. J. Jeffrey, and D. E. Knuth, "On the LambertW Function," Adv. Comput. Math., vol. 5, p. 329359, 1996.

[23] A. L. Moustakas, S. H. Simon, and A. M. Sengupta, "MIMO Capacity through Correlated Channels in the Presence of Correlated Interferers and Noise: A (not so) Large $N$ Analysis," IEEE Trans. Inf. Theory, vol. 49, no. 10, pp. 2545-2561, Oct. 2003.

[24] J. Wagner, B. Rankov, and A. Wittneben, "Large n Analysis of Amplifyand-Forward MIMO Relay Channels With Correlated Rayleigh Fading," IEEE Trans. Inf. Theory, vol. 54, no. 12, pp. 5735-5746, Dec. 2008. 